\title{
The Ethical Obligation to Vaccinate Children and Its Policy Implications
}

\author{
Johan C. Bester (University of Nevada)
}

\begin{abstract}
:
The vaccination of children has unique ethical implications. This article examines the ethics of childhood vaccination from two different perspectives. First, vaccination is approached as a preventive medical decision involving a child, using the ethical framework of medical decision-making for children. Second, vaccination is considered from a social and societal perspective, drawing on arguments from justice. From both these perspectives it is apparent that an ethical obligation exists to protect children against vaccine-preventable diseases by the appropriate use of vaccination. These ethical considerations have implications for policy, and in the paper a three-tiered approach to vaccination policy is defended: judicious use of mandatory policies, educational interventions, and building of trust.
\end{abstract}

Keywords:

immunization of children; justice; pediatric decision-making; vaccination ethics; vaccination policy

The vaccination of children has unique ethical implications for society, parents, and clinicians. I argue that childhood vaccination places moral claims on society, and in particular on those who stand in significant relationships with them. If we fail to provide vaccination, we wrong children. These ethical considerations have implications for how we think about vaccination and vaccination policy.

Vaccination is unique in that it inhabits two different ethical spheres. On one hand, it is a medical decision involving an individual child. On the other hand, vaccination is a societal endeavor with implications for public health and broader society. I will examine childhood vaccination from both these perspectives, and conclude that arguments grounded in these different perspectives ground a moral obligation to vaccinate children. From there I will briefly consider one or two policy implications of these ethical considerations.

\section{Decision-Making for the Individual Child}

In one sense, vaccination is a medical decision involving an individual child. Vaccination discussions and delivery often occurs during routine medical preventive care. A parent and a clinician have to decide whether to vaccinate this child as part of the preventive care of the child. When vaccine refusal happens, or if there are missed vaccination opportunities, this is often where it happens. These issues play out in preventive care visits, in clinics, in doctor's offices. It is therefore critical that we understand the ethical implications of medical decision-making for the child.
The framework for medical-decision making for children takes significant features and relationships of children into account (American Academy of Pediatrics, 2016; Bester, 2019a; McCullough, 2009). Young children lack the capacity to make their own medical decisions, and are dependent on others to protect their interests. Parents and clinicians have specific obligations in this regard. Parents have the authority to make medical decisions for their children, and have to make decisions for the child that protect and promote the welfare of the child. Clinicians also stand in a special relationship with the child, that of clinician-patient, and have obligations to promote and protect the interests of the child. Thus, during medical care of a child, parents and clinicians work together to find the best course of action to promote the health and welfare of the child.

The ethical standard at work here is the best interest standard, an ethical and legal principle that guides decisionmaking about children in many countries (Bester, 2019b; Pope, 2011). The best interest standard is a general ethical guideline that forms the basis of many policies and more specific obligations with regards to children, in medical decision-making specifically (Bester, 2019a; Bester, 2019b; Kopelman, 1997). It guides the obligations of parents to their child, and guides the obligations of clinicians to their childpatient. Basically stated, in every decision about a child the option should be chosen among available options that best protects the interests of the child. Interests refer to identifiable components of wellbeing of the child, so that if we promote a child's interests we promote that child's welfare, and if we act against a child's interests, we set back 
that child's welfare. Consequently, in all decisions about the child, it is the welfare of the child that is the central focus and the appropriate starting point.

When it comes to the decision to vaccinate, there are only two options: to vaccinate the child, or not vaccinate the child. According to the best interest standard, we must consider the implications of these two options on the welfare of the child. The right thing to do is the course of action that best promotes and protects the welfare of the child. Here it becomes important to consider the empirical attributes of vaccinations, weighing the benefits and risks associated with vaccination or with non-vaccination (Dawson, 2005). To simplify argumentation, I will focus on the empirical attributes of measles vaccine as example (Bester, 2016; Bester, 2017; Moss, 2017). But I should note that these arguments and conclusions will hold similarly for other vaccines that have somewhat similar attributes, such as vaccines against diphtheria, pertussis, polio, and others generally recommended for children (Centers for Disease Control and Prevention, 2015).

Measles is a serious and highly infectious disease (Bester, 2016; Bester, 2017; Moss, 2017). It has significant potential implications for the welfare of children. These include pneumonia, a variety of brain infections of inflammations, ear infection, diarrhea, malnutrition, and death. Each year, measles caused thousands of deaths, thousands of hospitalizations, and hundreds of thousands of cases prior to vaccine age in the United States alone. Vaccination is highly effective. One dose of measles vaccine gives immunity to $95 \%$ of children, and two doses to $99 \%$. The vaccine adverse effect rate compares favorably with other medical interventions. The adverse effects that occur more commonly are trivial and passing, and include things such as joint pain, rash, and fever. The most serious adverse effect is serious allergic reaction, which happens in less than 1 in a million doses. The adverse effect rate from vaccination compares favorably with other interventions that are often used for children, such as medicine for fever or transporting children by car. There are some children who cannot get the vaccine, because they have some medical contra-indication. They are too young, they are allergic, or they have immune compromise. Such children would not benefit from vaccination, or may suffer serious harm from vaccination. These children depend on high public vaccination rates, herd immunity, to protect them from measles.

From this perspective, it may be noted that the benefits of vaccination are substantial. A child derives reliable, life-long protection from a serious infectious disease. The adverse effect rate is low, negligible when compared to risk from disease, and compares favorably with other things children are often exposed to. Vaccination protects the welfare of a child in significant ways, and has to be viewed as the option supported by the best interest standard. The exception are those children who have a medical contra-indication to vaccination. Someone with allergy to a vaccine component may have a severe reaction to vaccination. A child with immune-compromise may develop serious adverse effects from measles vaccine. In these children with medical contraindications to vaccination, the risks from vaccination appear to outweigh the benefits, so that non-vaccination is the better option. We may therefore stipulate that it is ethically supported to provide vaccination for every child who does not have a medical contra-indication to vaccination. In children with known medical contra-indications (fortunately, a very small minority of children) it is ethically supported to withhold vaccination.

Perhaps, some may argue, there is a case for nonvaccination being the best for an individual child in a highly vaccinated society. This way, the child is protected against disease while not taking the risk of vaccine-related adverse effects, minimal though these risks may be. There are a number of ways to respond to this argument (Bester, 2017). First, this seems unfair to other children. It can be argued that the parent or clinician who reasons this way decides to freeride a public good, to use the efforts of others to establish herd immunity in a way that is unfair and ethically out of bounds. Second, direct vaccination is a much better protection than reliance on herd immunity. Even highly vaccinated societies suffer outbreaks that spread among chains of susceptible people. The actions of others may lead to loss of herd immunity, as has happened in some countries. If you get disease in a highly vaccinated country, the complication rate is higher than in countries with lower vaccination rates. Preventing disease requires avoidance of areas where infection may be likely, which limits the options available to the child, particularly in an outbreak. Third, the argument is self-defeating. If it really is better for such a child to forego vaccination, it would also be better for other children to forego vaccination. Each individual child considered would be left unvaccinated because of this argument. Ultimately herd immunity will be lost, and the welfare of these children will be at risk. If we try to protect the welfare of an individual child by appealing to this argument, we end up putting the welfare of the child at risk.

Even in highly vaccinated societies it is better for each eligible child to be vaccinated. This way the individual child is protected through direct vaccination, and those who cannot receive the vaccine enjoys some measure of protection through the establishment of herd immunity. Children ought to be vaccinated, unless there is a clear case that the risks would outweigh the benefits for a particular child.

\section{Justice and Society's Obligation to Children}

Vaccination is not just an individual medical decision. It is also a societal concern, something with justice implications for society and its members.

To see this point, we start off with the simple premise that a just society takes steps to protect the welfare of its children against preventable harms (Bester, 2018). This premise can be grounded by reference to various theories of justice or 
social morality. Most straight-forwardly, it can be grounded by reference to a wellbeing theory of justice, such as the social justice theory of Powers and Faden (2006). According to this theory, a just society ensures that its members reach a minimum level of wellbeing. Society must establish the kind of social environment that would allow its children to reach minimum levels of wellbeing, and must be on the lookout for systematic factors or influences that may lead people or groups to fall below minimal levels of wellbeing. In their theory, the health of children is of particular importance, as children are vulnerable and dependent, and ill-health during childhood lock in disadvantage and falling below acceptable levels of wellbeing for life. Vaccination in particular is of importance in this regard.

Other approaches to justice or social morality would also establish this premise. If we adopt a utilitarian perspective, we may see that a society that protects the welfare of its children against preventable harm has more people that fare better than a society that does not do so. From a Rawlsian contractarian justice perspective, we must ensure fair equality of opportunity, and we must ensure that the worstoff are in the best position they can possibly be; both of these principles direct us to take steps to protect the welfare of children against preventable harms. A capability theory of justice directs society to foster and protect the development of capabilities that would enable flourishing in its members, which means that the health of children must be protected against disease and injury that would limit the development of such capabilities. From each of these different perspectives we can see that it is justifiable to posit the simple truth that a just society protects the wellbeing of its children. Consider also that the implication of rejecting this premise is the acceptance of the rather unappetizing and hard-to-justify opposite: that a just society does not take steps to protect the wellbeing of its children against preventable harms. It is not clear how such a premise would be justified, or how one would feel comfortable with such a premise within one's system of morality.

When we start with the idea that justice asks of society to take steps to protect the welfare of children against preventable injuries and insults, one can see the ethical implications of vaccination directly (Bester, 2018). If we vaccinated children against disease in a way that protect children in so far as possible through direct vaccination, and protect those who cannot be vaccinated through herd immunity, we protect these children as best we can from the effects of vaccine-preventable disease on their wellbeing. The vaccine is safe and effective, and moreover results in significant savings in direct healthcare expenditure and indirect social costs. Here we see a highly effective step society can take to protect the wellbeing of countless children, in a way that pays for itself many times over. There can be no doubt that justice requires of a society to vaccinate its children.

This means that vaccination is something owed to children, a moral claim that children have on society. Justice asks of governments and healthcare institutions to institute safe and accessible vaccination programs, and to ensure access for all children to vaccination. Justice directs parents, guardians, and clinicians to make efforts to have children under their care vaccinated in a way that is safe and timely. Children who can be protected through direct vaccination, should be. Those who cannot receive the vaccine for a medical reason, should enjoy the measure of protection that herd immunity provides.

This line of argumentation removes vaccination from the sphere of parental or societal discretion. It is not optional; it is a moral obligation. Societies that fail to institute safe vaccination programs with easy access for children act unjustly. Clinicians, parents, or caregivers who place barriers before vaccination of children or remove the opportunity for children to be protected through vaccination act unjustly.

I have now considered two lines of moral argumentation that come to a similar conclusion: that there is an ethical obligation to protect children through vaccination, and that this represents a moral claim on behalf of the child upon society and those individuals who stand in significant care relationships with the child. We are speaking here of moral obligation, and the question of which policy measures are justified is a next question.

\section{Policy Implications of the Ethical Requirement to Vaccinate}

There are good ethical reasons to conclude that children ought to be vaccinated. This is supported both from the perspective of decision-making about the individual child, and from the perspective of what society owes children in general. A society ought to guarantee safe and timely access to vaccination for its children. This places obligations on the institutions and members of society that stand in positions to act in the interests of children. Most straight-forwardly, parents and clinicians are obligated to see to it that children under their care are vaccinated. Society must institute vaccination programs, a supply of vaccination, and equitable access for all to vaccination. This means government must step in to regulate and guarantee this supply, in co-ordination with industry and healthcare institutions.

One particular policy focus is parental vaccine refusal. If parents refuse vaccination and society must guarantee vaccination for children, society must respond to vaccine refusal. There are many reasons why parents refuse vaccines, and also different policy responses that may be followed (Horne et al., 2015; Navin et al., 2019; Omer et al., 2009). To my mind, there are three components to such a policy: mandatory measures, education, and building of trust relationships (Bester, 2015).

The ethical obligation to vaccinate provides a powerful justification for the use of compulsory vaccination policies where no other reason for non-vaccination exists than 
parental refusal. One must consider carefully what such a policy looks like. Compulsory vaccination policy makes use of state power to enforce vaccination, leveraging a penalty in cases of non-vaccination. Use of state power may have unforeseen impacts and costs, and care must be taken not to impose penalties that leave the children who are meant to be protected worse off. For example, heavy financial penalties may place families in precarious positions, and end up creating difficulty and burdens on welfare for the very children the policy is meant to protect. Another factor to consider is that stringent compulsory policies may foment backlash and polarize the issue of vaccination, which would be counter-productive to the goal of ensuring maximal vaccination uptake.

Compulsory policies have their place, and have the ability to increase vaccination uptake. Careful consideration of the competing values that should be protected must accompany the implementation of such a policy. In the many states in the United States, school attendance is used as lever to enforce mandatory vaccination, so that children who are unvaccinated are not able to attend a public school (Omer et al., 2009). It increases vaccination rates and overall seems to work acceptably well. But it can be argued that it withholds from unvaccinated children a good (public education) that may have implications for the wellbeing of the child over and above suffering the misfortune of being unvaccinated. Fortunately, there are generally other options for education for such children, and there is the additional consideration that such school-exclusion policies protect the wellbeing of those children who cannot receive vaccination and depend on high vaccination rates in their peers.
Education plays an important role in various ways and facets. Public messaging and educational interventions about vaccination and its effectiveness is key, and can remove vaccine hesitancy and vaccine refusal in some parents (Horne et al., 2015; Navin et al., 2019). However, it is clear that there are some people in whom education can backfire. Some research has shown that people with the strongest antivaccine sentiment can actually be fortified in their opposition to vaccines by exposure to educational interventions on the safety and effectiveness of vaccinations. For this reason, public educational interventions should carefully be studied to see the effect on intended audience, and such education should be employed where it is shown to be effective. Ultimately, there may be some parents who will not be persuaded, in whom anti-vaccine sentiment is a part of their identity and in whom educational interventions will strengthen anti-vaccine sentiment. The children of such parents are morally entitled to protection against disease through vaccination, and may well be dependent on some sort of compulsory policy to get it.

Lastly, the trust of parents and the general public in vaccinations is important (Bester, 2015). If people trust in vaccinations, vaccination will happen. If people distrust vaccination, not even the best policy in the world can ensure sufficient vaccination uptake. Any policy intervention must address this trust gap. One way to do this is to ensure the building of trust relationships between clinicians who care for children and parents. This should be a central feature of any policy that focuses on the health of children more generally, and on the uptake of vaccinations in particular.

\section{References}

American Academy of Pediatrics, Committee on Bioethics. (2016). Informed consent in decision-making in pediatric practice. Pediatrics, 138(2). https://doi.org/10.1542/peds.2016-1485

Bester, J. C. (2016). Measles and measles vaccination: A review. JAMA Pediatrics, 170(12), 1209-1215. https://doi.org/10.1001/jamapediatrics.2016.1787

Bester, J. C. (2017). Measles vaccination is best for children: The argument for relying on herd immunity fails. Journal of Bioethical Inquiry, 14(3), 375-384. https://doi.org/10.1007/s11673-017-9799-4

Bester, J. C. (2018). Not a matter of parental choice but of social justice obligation: Children are owed measles vaccination. Bioethics, 32(9), 611-619. https://doi.org/10.1111/bioe.12511

Bester, J. C. (2019a). The best interest standard and children: Clarifying a concept and responding to its critics. Journal of Medical Ethics, 45(2), 117-124. https://doi.org/10.1136/medethics-2018-105036

Bester, J. C. (2019b). The best interest standard is the best we have: Why the harm principle and constrained parental autonomy cannot replace the best interest standard in pediatric ethics. The Journal of Clinical Ethics, 30(3), 223231 .

Centers for Disease Control and Prevention. (2015). Epidemiology of vaccine-preventable diseases. Public Health Foundation. https://www.cdc.gov/vaccines/pubs/pinkbook/index.html

Dawson, A. (2005). The determination of 'best interests' in relation to childhood vaccinations. Bioethics, 19(2), $72-89$. https://doi.org/10.1111/j.1467-8519.2005.00425.x 
Horne, Z., Powell, D., Hummel, J. E., \& Holyoak K. J. (2015). Countering antivaccination attitudes. Proceedings of the National Academy of Sciences of the United States of America, 112(33), 10321-10324. https://doi.org/10.1073/pnas.1504019112

Kopelman, L. M. (1997). The best-interests standard as threshold, ideal, and standard of reasonableness. Journal of Medicine and Philosophy, 22(3), 271-289. https://doi.org/10.1093/jmp/22.3.271

McCullough, L. B. (2009). Contributions of ethical theory to pediatric ethics: Pediatricians and parents as co-fiduciaries of pediatric patients. In G. Miller (Ed.), Pediatric Bioethics (pp. 11-21). Cambridge University Press.

Moss, W. J. (2017). Measles. The Lancet, 390(10111), 2490-2502. https://doi.org/10.1016/S0140-6736(17)31463-0

Navin, M. C., Wasserman, J. A., Ahmad, M., \& Bies, S. (2019). Vaccine education, reasons for refusal, and vaccination behavior. American Journal of Preventive Medicine, 56(3), 359-367. https://doi.org/10.1016/j.amepre.2018.10.024

Omer, S. B., Salmon, D. A., Orenstein, W. A., deHart, M. P., \& Halsey, N. (2009). Vaccine refusal, mandatory immunization, and the risks of vaccine-preventable diseases. New England Journal of Medicine, 360(19), 19811988. https://doi.org/10.1056/NEJMsa0806477

Pope, T. M. (2011). The best interest standard: Both guide and limit to medical decision making on behalf of incapacitated patients. The Journal of Clinical Ethics, 22(2), 134-138.

Powers, M., \& Faden, R. R. (2008). Social justice: The moral foundations of public health and health policy. Oxford University Press.

\section{Recommended Citation}

Bester, J. C. (2020). The ethical obligation to vaccinate children and its policy implications. On Education. Journal for Research and Debate, 3(8). https://doi.org/10.17899/on_ed.2020.8.2

\footnotetext{
About the Author

Johan C. Bester is assistant professor and director of bioethics at the department of medicine of the University of Nevada, Las Vegas.
} 